\title{
Effects of custom-made insoles on idiopathic pes cavus foot during walking
}

\author{
Jung-Kyu Choi ${ }^{\mathrm{a}}$, Eun-Jong Cha ${ }^{\mathrm{b}}$, Kyung-Ah Kim ${ }^{\mathrm{b}}$, Yonggwan Won ${ }^{\mathrm{c}}$ and Jung-Ja Kim ${ }^{\mathrm{d}, \mathrm{e},{ }^{*}}$ \\ ${ }^{a}$ Department of Healthcare Engineering, Chonbuk National University, Jeonju, South Korea \\ ${ }^{b}$ College of Medicine, Chungbuk National University, Cheongju, South Korea \\ ${ }^{c}$ School of Electronics and Computer Engineering, Chonnam National University, Gwangju, South \\ Korea \\ ${ }^{d}$ Division of Biomedical Engineering, Chonbuk National University, Jeonju, South Korea \\ ${ }^{e}$ Research Center of Healthcare \& Welfare Instrument for the Aged, Chonbuk National University, \\ Jeonju, South Korea
}

\begin{abstract}
From a subject group of pes cavus, the purpose of this study was to evaluate the biomechanical characteristics of lower limbs, based on plantar foot pressure and electromyography (EMG) activities, by the effects on two kind of custommade insoles. Ten individuals among thirty females with a clinical diagnosis of idiopathic pes cavus (mean age (SD): 22.3 (0.08) years) were selected for the study. The plantar foot pressure data and EMG activities of four lower limb muscles were collected, when subjects walked on a treadmill, under three different experimental conditions. The plantar foot pressure data was analyzed, after the bilateral foot was divided into three areas of masks and into four sections of stance phase, to compare plantar foot pressure. The EMG activities were analyzed for integrated EMG (IEMG) value. The results show that plantar foot pressure concentrated in particular parts is decreased by custom-made insoles. In the case of EMG, all the muscle activities decreased significantly. The custom-made insoles dispersed pressure concentrated by the higher medial longitudinal arch and improved the efficient use of muscles. In particular, the extension structure in the forefoot of custom-made insoles was more efficient for pes cavus. Therefore, it could help patients to walk, by offering support to prevent the disease of pes cavus deformity, and to relieve the burden and fatigue in the lower limbs on gait.
\end{abstract}

Keywords: Gait, custom-made insole, pes cavus, plantar foot pressure, EMG

\section{Introduction}

Foot is the most important element in the standing posture and bipedalism of the human body. Foot, with only $5 \%$ of the entire surface on the body, supports the body weight of $95 \%$, and has the function of absorbing the impact from the ground $[1,2]$.

The meaning of locomotion is just motion to move from one position to another, and the gait is a special motion to work a combination of the feet, legs and waist, during moving the human body from one point to another [3]. Bipedalism, including walking, running and jumping, is the most natural human motion, and the default behavior that anyone can easily perform, if they have a normal body [4].

\footnotetext{
*Address for correspondence: Jung-Ja Kim, Division of Biomedical Engineering, Chonbuk National University, Jeonju, South Korea. Tel.: +82-63-270-4102; Fax:+82-63-270-2247; E-mail: jungjakim@jbnu.ac.kr.
} 
However, the complex coordination of different skeletal muscles and the nervous system make the human individual have a high amount of balancing and stability on gait. When the body moves forward, one of the lower limbs maintains stability by supporting the weight in the stance phase during each lower limb step [5]. When a human walks for $1 \mathrm{~km}$, there is about a $15 \mathrm{t}$ weight increase on the foot, and the pressure that occurs by weight or the push-off exercise causes soft tissue strain or stress effects on the human body [6].

The pes cavus, frequently known as the "high-arched foot" or "cavoid foot", is a medical condition, in which the medial longitudinal arch (MLA) has the height of the foot raised, and accepted to be rigid structurally, and runs a complicated deformity, to cause the equinus of the forefoot, or the varus of the hindfoot $[7,8]$. This foot condition occurs bilaterally in $8-15 \%$ of the population [9]. It is known to be commonly caused by high-heel, or diseases like Charcot-Marie-Tooth and polio that transform the musculoskeletal system and bring excessive internal rotation of the ankle and knee joint or constricted muscles, but most of the patients with pes cavus are expected to have idiopathic pes cavus [10]. As a consequence, the contact area of the foot on the floor is narrowed, while the ankle or heel is tilted out exteriorly. Patients who have mild form often exhibit no symptoms. However, patients with an advanced disease feel fatigue on walking easily, and frequently complain of oppressive pain in the metatarsal heads [11-15]. Over time, the mechanical overloading by the raised MLA adversely affects the balance of the body, and causes diseases, such as plantar fasciitis, metatarsalgia, sesamoiditis and asymmetry of the pelvis [16-18].

Pes cavus deformities are treated using surgical method or orthoses method like custom-made insoles $[19,20]$. Custom-made insoles using the non-invasive method are produced to fit the patient's foot, and are defined as external devices applied to a body segment in order to prevent or correct dysfunction (mobility limitation, correction or prevention of vicious positions or deformations, and reduction of the axial load) [21]. They control movement of the abnormal foot, and reduce the symptoms of diseases like fatigue and pain. They are also used for correcting excessive or undesired movement. According to previous studies on the effect of custom-made insoles, the results of several research show differences in measurement factors. The changes analyzed in the peak vertical force and maximum vertical loading rate in a group wearing shoes with four different types of insoles showed that the different insoles had no appreciable effect on the values measured [22]. The separate insoles also resulted in no significant differences in the calcaneal eversion, maximum pronation, and total pronation of the foot [23]. However, custom-made insoles usually prevent deformity and necrosis of the foot by dispersion of the pressure at the forefoot, where an ulcer is caused on diabetes patients [24-26]. When insoles were equipped with, the plantar foot pressure was reduced by $30-40 \%$ in the area of the first metatarsal head and medial calcaneus and increased by $5-10 \%$ in the total contact area [27]. It also reduces the maximum pressure by $37 \%$ in the heel and $27 \%$ in the forefoot so that the ground reaction is absorbed [28]. Kang et al. informed that pain of the forefoot, especially the metatarsal was caused by excessive pressure load, and it was relieved after wearing insoles with a metatarsal pad for 2 weeks. The result of this study also showed that the plantar pressure was distributed [29]. Mueller et al. noted that the integral pressure-time and maximum planar pressure were reduced by $16-24 \%$ on metatarsal heads when total-contact insoles were equipped to control abnormal movement of feet and disperse excessive plantar pressure, and in addition, by $29-47 \%$, when total-contact insoles with metatarsal pad were equipped to disperse pressure of the forefoot [30]. It was reported that custom-made insoles had distributed the concentrated pressure of a specific area to the whole of the foot, and relieved impact, and pain by high pressure [31].

Until now, studies about the gait characteristic on the effect of custom-made insoles have been carried out, but more accurate study is necessary because there are still various variables, such as a varie- 
ty of diseases of the foot and the form of insoles. In most of the previous research, the effect of custom-made insoles is studied from the viewpoint of pressure distribution. However, the muscles activity on lower limbs based on mechanical movement was not investigated during walking. Accordingly, the purpose of this study was to provide information of biomechanical gait characteristics by analyzing the influence of custom-made insoles on plantar foot pressure and muscle activity.

\section{Methods}

\subsection{Subjects}

The study was performed for ten persons who had received definite diagnosis of pes cavus by a podiatrist among 30 females suspected of pes cavus (age $22.3 \pm 0.08$ years, height $159.9 \pm 2.2 \mathrm{~cm}$, weight $50.8 \pm 3.69 \mathrm{~kg}$, foot size $237.9 \pm 3.27 \mathrm{~mm}$, mean $\pm \mathrm{SD}$ ). All subjects had no history of injury or disease except pes cavus in the musculoskeletal system of the lower extremities. An ethical approval was obtained from the Human Ethics Committee of Chonbuk National University Medical School, and information about this study of purpose and procedure were provided to the subjects, who signed the test consent form.

\subsection{Tools}

In this study, the custom-made insoles were produced on shell structures of $2 / 3$ length insoles, which is the basic structure in the orthoses method, and full length insoles to investigate the effect of front structure, as shown in Figures 1(a) and 1(b). They were made by prescriptions of podiatrist to reduce supination of the foot for each subject, and molded with the insertion of metatarsal pads to distribute the pressure concentrated in the forefoot area and structured to reduce heel tilted out to the exterior [32]. The shell, metatarsal pad and surface of the custom-made insoles were composed of polypropylene, polyurethane and artificial leather, respectively, as shown in Figures 1(a) and 1(b). The shoes selected were common running- shoes, and custom-made insoles were suitably inserted in the shoes, as shown in Figure 1(c).

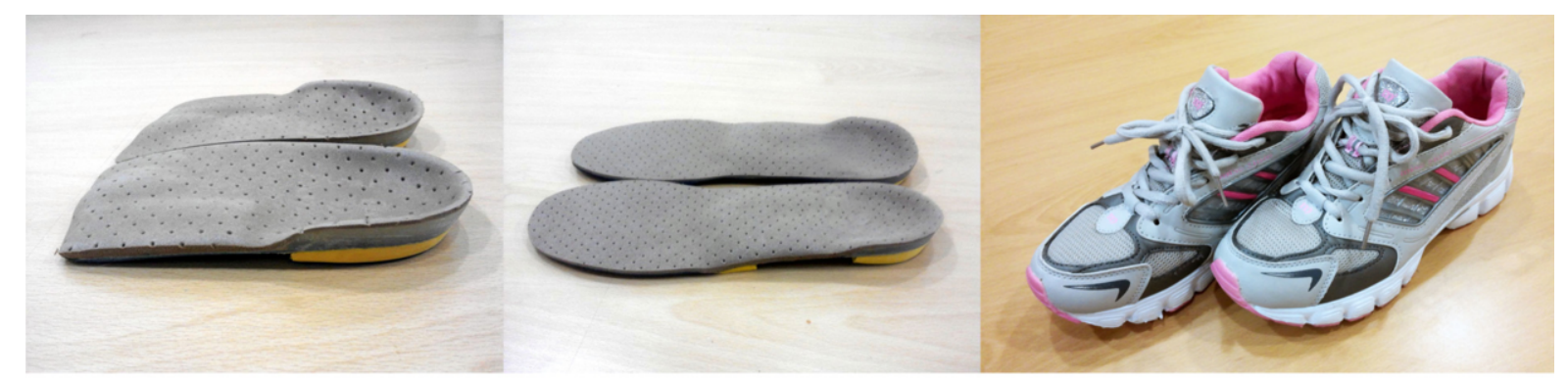

(a) (b) (c)

Fig. 1. (a) 2/3 Length insoles. (b) full length insoles. (c) normal shoes. 


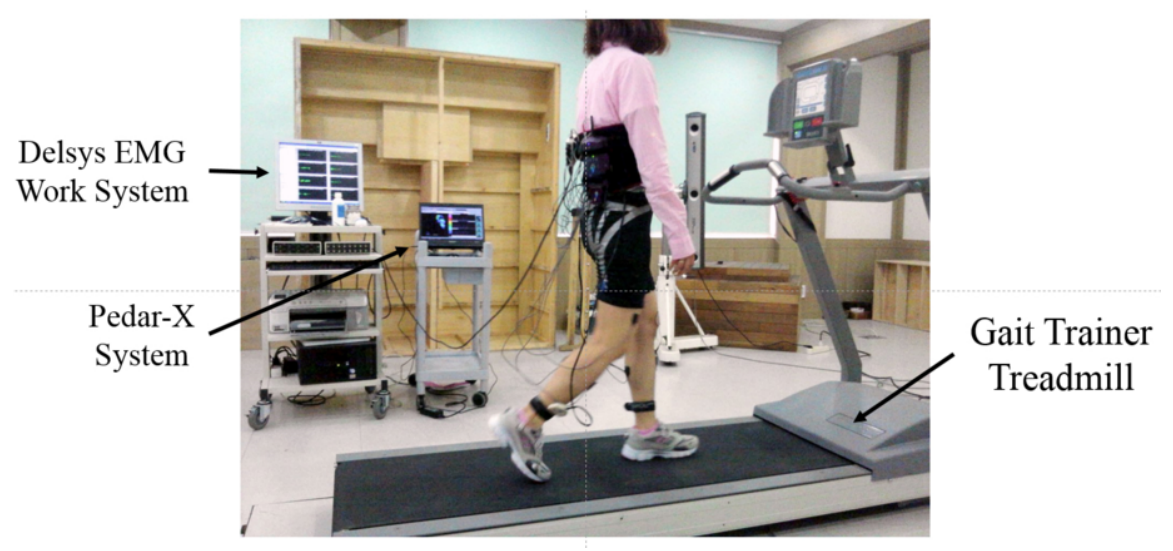

Fig. 2. Experiment process.

\subsection{Study procedure}

All subjects walked on the Gait Trainer Treadmill (Biodex, New York, USA) under three conditions: walking with normal shoes (NS), walking with $2 / 3$ length custom-made insoles in normal shoes (CI) and walking with full length custom-made insoles in normal shoes (FCI). For comparative analysis of data from conditions, each subject was asked to walk five times on a treadmill for 1 minute at a step speed of $3.0 \mathrm{~km} / \mathrm{h}$ in reference to $1.08 \mathrm{~m} / \mathrm{s}$ that was the average woman step speed on Korea [33]. Before the experiment, subjects walked for five minutes to adapt to gait on a treadmill and took rest for 5 minutes to prevent fatigue in between experiments, as shown in Figure 2.

\subsection{Data Acquisition}

Plantar foot pressure and electromyography (EMG), a research tool to measure the amplitude of muscle activation were measured to analyze the effect of custom-made insoles on the gait [34]. Distributions of plantar foot pressure were measured by using the Pedar-X system (Novel Gmbh, Munich, Germany). Each insole of the system was composed of 99 capacitive sensors (sample-rate $100 \mathrm{~Hz}$ ) and data were transmitted and recorded by using a Bluetooth connection to a computer. Muscle activities were recorded by using the Delsys EMG Work system (Delsys Inc., Boston, USA), which was able to collect data through eight channels. The tibialis anterior and medial gastrocnemius were involved in dorsiflexion and plantar flexion of the ankle joint, respectively; and the rectus femoris and musculus biceps femoris were deeply related to movement of the hip and knee joint, respectively, on gait [5]. DE-3.1 Surface Electrodes (Delsys Inc., Boston, USA) were therefore attached to the rectus femoris (RF), the tibialis anterior (TA), the musculus biceps femoris (MBF), and medial gastrocnemius (MG) of both legs, as shown in Figure $3[35,36]$.

\subsection{Data Analysis}

The data of contact area, maximum force, peak pressure and mean pressure were analyzed to compare the plantar foot pressure data collected from each condition after the foot was divided into three areas of masks (forefoot, midfoot and hindfoot). The data were also divided into four steps of the stance phase: initial contact (0-4\%), loading response (0-25\%), mid-stance (25-75\%) and terminal stance $(75-100 \%)$. The masks were defined, and the data were analyzed by using Pedar-X Analyze 

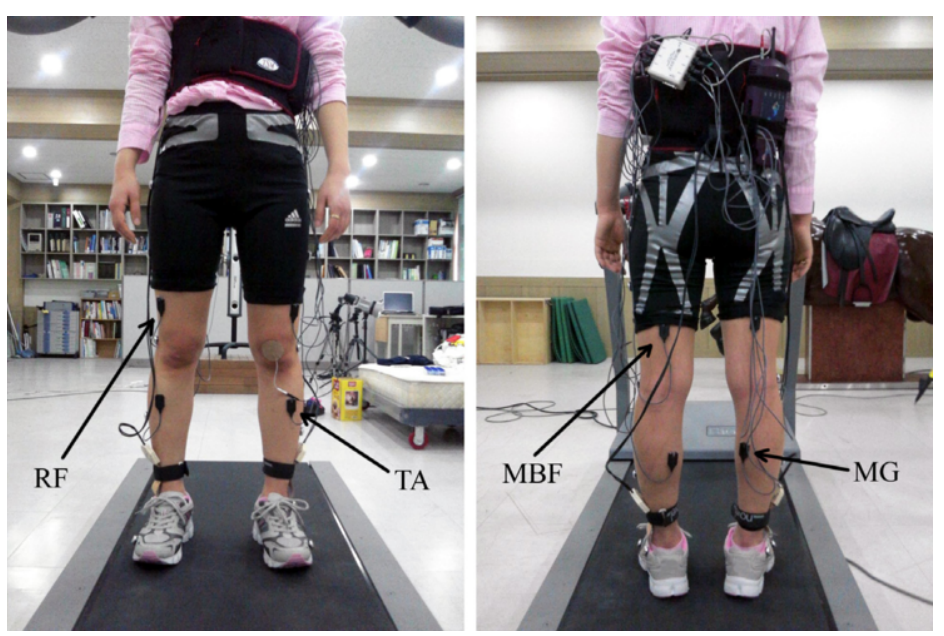

Fig. 3. Measurement of EMG.

Software (Novel Gmbh., Munich, Germany). EMG signals measured from the surface electrodes attached on each muscle were filtered by bandpass filter (passband $20-450 \mathrm{~Hz}$ ), and sampled at $1,000 \mathrm{~Hz}$, to reduce EMG noise for collecting accurate data. The muscle activities were analyzed into the Integrated EMG (IEMG) value.

Table 1

The results of plantar foot pressure in masks

\begin{tabular}{|c|c|c|c|c|}
\hline & & $\begin{array}{l}\text { Normal Shoes } \\
\text { (NS) }\end{array}$ & $\begin{array}{l}2 / 3 \text { length Custom-made } \\
\text { Insoles }(\mathrm{CI})\end{array}$ & $\begin{array}{l}\text { Full length Custom-made } \\
\text { Insoles (FCI) }\end{array}$ \\
\hline \multirow{3}{*}{$\begin{array}{l}\text { Contact Area } \\
\left(\mathrm{cm}^{2}\right)\end{array}$} & Forefoot & $40.24 \pm 4.23$ & $38.17 \pm 4.79^{*}$ & $31.75 \pm 3.84^{* *, \alpha}$ \\
\hline & Midfoot & $30.21 \pm 5.77$ & $38.45 \pm 5.16^{*}$ & $47.36 \pm 4.87^{* *, \alpha}$ \\
\hline & Hindfoot & $32.44 \pm 1.23$ & $31.48 \pm 1.95^{*}$ & $29.23 \pm 1.46^{* *, \mathrm{p}}$ \\
\hline \multirow{3}{*}{$\begin{array}{l}\text { Maximum } \\
\text { Force }(\mathrm{N})\end{array}$} & Forefoot & $312.58 \pm 49.20$ & $296.83 \pm 53.79^{*}$ & $216.87 \pm 57.39^{* *, \alpha}$ \\
\hline & Midfoot & $133.87 \pm 37.8$ & $236.39 \pm 58.49^{*}$ & $246.87 \pm 59.17^{* *, \alpha}$ \\
\hline & Hindfoot & $301.66 \pm 54.21$ & $283.29 \pm 48.36^{*}$ & $222.84 \pm 45.26^{* *, \alpha}$ \\
\hline \multirow{3}{*}{$\begin{array}{l}\text { Peak Pressure } \\
(\mathrm{kPa})\end{array}$} & Forefoot & $171.09 \pm 34.9$ & $179.06 \pm 49.4^{*}$ & $142.34 \pm 31.5^{* *, a}$ \\
\hline & Midfoot & $116.98 \pm 48.33$ & $130.8 \pm 35.01^{*}$ & $149.92 \pm 21.75^{* *, \alpha}$ \\
\hline & Hindfoot & $153.5 \pm 22.53$ & $151.73 \pm 27.48^{*}$ & $122.47 \pm 24.98^{* *, \alpha}$ \\
\hline \multirow{3}{*}{$\begin{array}{l}\text { Mean Pressure } \\
(\mathrm{kPa})\end{array}$} & Forefoot & $82.51 \pm 10.81$ & $82.39 \pm 13.21$ & $70.83 \pm 12.31^{* *, \alpha}$ \\
\hline & Midfoot & $52.99 \pm 10.83$ & $65.67 \pm 11.13^{*}$ & $58 \pm 10.07^{* *, \alpha}$ \\
\hline & Hindfoot & $94.36 \pm 15.97$ & $92.84 \pm 16.14^{*}$ & $77.26 \pm 15.21^{* *, \alpha}$ \\
\hline
\end{tabular}

Note: ${ }^{*}: \mathrm{p}<0.05$ significant difference between NS and CI.

${ }^{* *}: \mathrm{p}<0.05$ significant difference between NS and FCI.

${ }^{a}: \mathrm{p}<0.05$ significant difference between $\mathrm{CI}$ and FCI. 


\subsection{Statistical Analysis}

The data, as plantar foot pressure and muscle activities from each condition, were analyzed by using SPSS 18.0 statistical software (SPSS Inc., Chicago, USA). A one-way ANOVA was performed to compare between data, and the statistical significance was determined at $p<0.05$ level with the Scheffe test.

\section{Results}

In this study, pressure distribution of the foot obtained from each condition was analyzed on contact area, maximum force, peak pressure and mean pressure, for three areas (forefoot, midfoot and hindfoot), as shown in Table 1 . The contact area significantly decreased by $2.63 \%$ and $1.5 \%$ in forefoot and hindfoot, respectively, and significantly increased by $12 \%$ in midfoot on CI condition, as against NS condition. In the case of FCI condition, it significantly decreased by $11.79 \%$ and $5.21 \%$ in forefoot and hindfoot, respectively, and significantly increased by $22.1 \%$ in midfoot. In comparison with CI condition, it significantly decreased by $9.61 \%$ and $3.71 \%$, respectively, in forefoot and hindfoot, and significantly increased by $10.37 \%$ in midfoot on FCI condition. The maximum force also significantly decreased by $2.58 \%$ and $3.14 \%$ in forefoot and hindfoot, respectively, and significantly increased by $27.69 \%$ in midfoot on CI condition as against NS condition. In the case of FCI condition, it significantly decreased by $18.08 \%$ and $15.03 \%$ in forefoot and hindfoot, respectively, and significantly increased by $29.68 \%$ in midfoot. In comparison with CI condition, it significantly decreased by $15.57 \%$ and $11.94 \%$ in forefoot and hindfoot, respectively, and significantly increased by $2.17 \%$ in midfoot on FCI condition. According to the result of the peak pressure, it significantly decreased by $0.58 \%$ in hindfoot and significantly increased by $2.28 \%$ and $5.58 \%$ in forefoot and midfoot respectively on CI condition, as against NS condition. In the case of FCI condition, it significantly decreased by $9.17 \%$ and $11.25 \%$ in forefoot and hindfoot, respectively, and significantly increased by $12.34 \%$ in midfoot. In comparison with CI condition, it significantly decreased by $11.43 \%$ and $10.67 \%$ in forefoot and hindfoot, respectively, and significantly increased by $6.81 \%$ in midfoot on FCI condition. The mean pressure significantly decreased by $0.07 \%$ and $0.81 \%$ in forefoot and hindfoot, respectively, and significantly increased by $10.69 \%$ in midfoot on CI condition, as against NS condition. A statistical significance difference was not found in the forefoot. In the case of FCI condition, it significantly decreased by $7.61 \%$ and $9.95 \%$ in forefoot and hindfoot, respectively, and significantly increased by $4.51 \%$ in midfoot. In comparison with CI condition, it significantly decreased by $7.54 \%, 6.2 \%$ and $9.15 \%$ in forefoot, midfoot and hindfoot, respectively, on FCI condition.

The distribution of plantar foot pressure was analyzed with four sections (initial contact, loading response, mid stance and terminal stance) on stance phase, as shown in Table 2. The contact area increased by $4.86 \%, 0.48 \%$ and $9.46 \%$ in initial contact, mid stance and terminal stance, respectively, and decreased by $1.54 \%$ in loading response on CI condition, as against NS condition. In the case of FCI condition, it increased by $9.06 \%$ in terminal stance, and decreased by $0.61 \%, 2.84 \%$ and $0.69 \%$ in initial contact, loading response and mid stance, respectively. In comparison with CI condition, it decreased by $5.48 \%, 1.3 \%, 1.18 \%, 0.41 \%$ in initial contact, loading response, mid stance and terminal stance, respectively, on FCI condition. Statistical significance differences were found in terminal stance on NS-CI and NS-FCI. The maximum force significantly decreased by $1.82 \%$ and $2.2 \%$ in initial contact and loading response, respectively, and significantly increased by $3.05 \%$ and $8.36 \%$ in mid stance and terminal stance, respectively, on CI condition, as against NS condition. A statistical signifi- 
Table 2

The results of plantar foot pressure in stance phase

\begin{tabular}{|c|c|c|c|c|}
\hline & & $\begin{array}{l}\text { Normal Shoes } \\
\text { (NS) }\end{array}$ & $\begin{array}{l}2 / 3 \text { length Custom- } \\
\text { made Insoles }(\mathrm{CI})\end{array}$ & $\begin{array}{l}\text { Full length Custom- } \\
\text { made Insoles (FCI) }\end{array}$ \\
\hline \multirow{4}{*}{$\begin{array}{l}\text { Contact Area } \\
\left(\mathrm{cm}^{2}\right)\end{array}$} & Initial Contact & $15.82 \pm 9.94$ & $17.43 \pm 10.86$ & $15.62 \pm 10.78$ \\
\hline & Loading Response & $42.25 \pm 17.92$ & $40.97 \pm 15.8$ & $39.9 \pm 13.7$ \\
\hline & Mid Stance & $74.18 \pm 4.21$ & $74.9 \pm 6.46$ & $73.15 \pm 5.79$ \\
\hline & Terminal Stance & $47.17 \pm 14.32$ & $57.03 \pm 16.82^{*}$ & $56.57 \pm 15.25^{* *}$ \\
\hline \multirow{4}{*}{$\begin{array}{l}\text { Maximum Force } \\
(\mathrm{N})\end{array}$} & Initial Contact & $55.65 \pm 44.15$ & $53.66 \pm 38.64$ & $47.18 \pm 44.65^{* *, \alpha}$ \\
\hline & Loading Response & $254.15 \pm 125.91$ & $243.16 \pm 123.3^{*}$ & $196.35 \pm 120.7^{* *, \alpha}$ \\
\hline & Mid Stance & $400.55 \pm 11.06$ & $425.76 \pm 31.47^{*}$ & $355.41 \pm 38.87^{* *, \alpha}$ \\
\hline & Terminal Stance & $283.42 \pm 128.78$ & $335.14 \pm 155.53^{*}$ & $271.19 \pm 156.3^{* *, \alpha}$ \\
\hline \multirow{4}{*}{$\begin{array}{l}\text { Peak Pressure } \\
(\mathrm{kPa})\end{array}$} & Initial Contact & $41.5 \pm 28.26$ & $36.75 \pm 24.59^{*}$ & $34.12 \pm 28.71^{* *, \alpha}$ \\
\hline & Loading Response & $115.07 \pm 41.63$ & $112.14 \pm 43.7^{*}$ & $90.18 \pm 36.38^{* *, \alpha}$ \\
\hline & Mid Stance & $127.05 \pm 17.04$ & $139.61 \pm 13.73^{*}$ & $121.61 \pm 19.58^{* *, a}$ \\
\hline & Terminal Stance & $128.97 \pm 45.55$ & $143.57 \pm 50.71^{*}$ & $120.07 \pm 41.37^{* *, \alpha}$ \\
\hline \multirow{4}{*}{$\begin{array}{l}\text { Mean Pressure } \\
(\mathrm{kPa})\end{array}$} & Initial Contact & $25.51 \pm 16.58$ & $22.75 \pm 14.61^{*}$ & $21.87 \pm 13.69^{* *}$ \\
\hline & Loading Response & $55.42 \pm 16.72$ & $54.27 \pm 17.74$ & $45.51 \pm 16.15^{* *, \mathrm{a}}$ \\
\hline & Mid Stance & $54.84 \pm 4.83$ & $57.71 \pm 4.63^{*}$ & $49.48 \pm 5.01^{* *, \alpha}$ \\
\hline & Terminal Stance & $56.45 \pm 15.02$ & $54.83 \pm 15.45^{*}$ & $49.63 \pm 15.24^{* *, \alpha}$ \\
\hline
\end{tabular}

Note: ${ }^{*}: \mathrm{p}<0.05$ significant difference between NS and CI.

${ }^{* *}: \mathrm{p}<0.05$ significant difference between NS and FCI.

$: \mathrm{p}<0.05$ significant difference between CI and FCI.

cance difference was not found in initial contact. In the case of FCI condition, it significantly decreased by $8.24 \%, 12.83 \%, 5.97 \%$ and $2.2 \%$ in initial contact, loading response, mid stance, and terminal stance respectively. In comparison with CI condition, it significantly decreased by $6.43 \%$, $10.65 \%, 9 \%$ and $6.04 \%$ in initial contact, loading response, mid stance and terminal stance, respectively, on FCI condition. According to the results of the peak pressure, it significantly decreased by $6.08 \%$ and $1.29 \%$ in initial contact and loading response, respectively and significantly increased by $4.71 \%$ and $5.35 \%$ in mid stance and terminal stance, respectively, on CI condition, as against NS condition. In the case of FCI condition, it significantly decreased by $9.76 \%, 12.12 \%, 2.19 \%$ and $3.57 \%$ in initial contact, loading response, mid stance and terminal stance, respectively. In comparison with CI condition, it significantly decreased by $3.7 \%, 10.85 \%, 6.89 \%$ and $8.91 \%$ in initial contact, loading response, mid stance and terminal stance, respectively, on FCI condition. The mean pressure significantly decreased by $5.73 \%, 1.05 \%$ and $1.46 \%$ in initial contact, loading response and terminal stance, respectively, and significantly increased by $2.57 \%$ in mid stance on CI condition, as against NS condition. A statistical significance difference was not found in loading response. In the case of FCI condition, it significantly decreased by $7.75 \%, 9.82 \%, 5.13 \%$ and $6.43 \%$ in initial contact, loading response and 
Table 3

The result of intergrated EMG (Vrms*sec)

\begin{tabular}{|c|c|c|c|c|}
\hline & $\begin{array}{l}\text { Rectus Femoris } \\
(\mathrm{RF})\end{array}$ & $\begin{array}{l}\text { Tibialis Anterior } \\
\text { (TA) }\end{array}$ & $\begin{array}{l}\text { Musculus Biceps } \\
\text { Femoris (MBF) }\end{array}$ & $\begin{array}{l}\text { Medial Gastroc-nemius } \\
(\mathrm{MG})\end{array}$ \\
\hline $\begin{array}{l}\text { Normal Shoes } \\
\text { (NS) }\end{array}$ & $0.00555 \pm 0.00064$ & $0.01789 \pm 0.00174$ & $0.00571 \pm 0.00046$ & $0.01557 \pm 0.00164$ \\
\hline $\begin{array}{l}2 / 3 \text { length Custom- } \\
\text { made Insoles }(\mathrm{CI})\end{array}$ & $0.00537 \pm 0.00059^{*}$ & $0.01599 \pm 0.00156^{*}$ & $0.00538 \pm 0.00048^{*}$ & $0.01369 \pm 0.00168^{*}$ \\
\hline $\begin{array}{l}\text { Full length Custom- } \\
\text { made Insoles (FCI) }\end{array}$ & $0.00536 \pm 0.00058^{* *}$ & $0.01609 \pm 0.00157^{* *}$ & $0.00537 \pm 0.00046^{* *}$ & $0.01373 \pm 0.00169^{* *}$ \\
\hline
\end{tabular}

terminal stance, respectively. In comparison with CI condition, it significantly decreased by $2.04 \%$, $8.77 \%, 7.68 \%$ and $4.97 \%$ in initial contact, loading response and terminal stance, respectively, on FCI condition. A statistical significance difference was not found in initial contact.

In results of the EMG, muscle activities significantly decreased by $1.74 \%, 5.61 \%, 3.01 \%$ and $6.38 \%$ in the RF, TA, MBF and MG, respectively, on CI condition, as against NS condition. In the case of FCI, it significantly decreased $1.75 \%, 5.32 \%, 3.12 \%$ and $6.25 \%$ in the RF, TA, MBF and MG, respectively. In comparison with the CI condition, statistical significance differences were not found in all of the regions on FCI condition, as shown in Table 3.

\section{Conclusion}

In this study, we evaluated the biomechanical characteristics of the lower extremities on gait of 10 females with shoes and two kinds of custom-made insoles, manufactured to relieve pes cavus deformities. The results of comparative analysis on the distribution of plantar foot pressure showed that it was increased by weight concentrated on the forefoot and hindfoot in pes cavus foot. When subjects walked on CI condition, the contact area, maximum force, peak pressure and mean pressure in the midfoot were significantly increased by the effect of structural characteristics to reduce the heel tilted out exteriorly, and metatarsal pads to distribute plantar foot pressure in the forefoot. As a result, the contact area, maximum force, peak pressure and mean pressure in the hindfoot were significantly decreased. These results were similar to research of Jung et al. and Chen et al. that study custom-made insoles made for pressure concentrated on particular areas [31,37]. The contact area and maximum force also decreased significantly in the forefoot by metatarsal pad as results of Mueller et al. [30]. However, the peak pressure increased against contact area and maximum force, and the mean pressure had no statistical significant difference in the forefoot. These results were judged by the effect of the 2/3 length structure on the CI condition, which contacted only the midfoot and hindfoot. In the case of the FCI condition, the contact area decreased in the forefoot and hindfoot, by the contact area of the midfoot increasing more than the CI condition. As a result, we were able to check that the maximum force, peak pressure and mean pressure decreased in the forefoot and hindfoot, while the maximum force and peak pressure increased in the midfoot. In comparison with the CI condition, pressure con- 
centrated in the forefoot was highly distributed by extension structure added through custom-made insoles, which influenced the hindfoot. From the viewpoint of the four sections on stance phases, the maximum force, peak pressure and mean pressure decreased in initial contact and loading response on the CI condition. This showed that the weight was dispersed by custom-made insoles, during progress from hindfoot to midfoot. In mid stance, the maximum force, peak pressure and mean pressure increased on the CI condition. This change was also the result due to the contact area increasing in the midfoot, by structural characteristics of the insoles. However, there was a difference that the maximum force and peak pressure increased, but the mean pressure decreased in terminal stance. This difference was also affected by the $2 / 3$ length structure of the CI condition at toe off, on the stance phase. According to the FCI condition, the maximum force, peak pressure and mean pressure decreased in all of the sections. As a result, we were able to conclude that there was effect by a difference between structures in the forefoot of two insoles.

The results of EMG analysis showed that it decreased significantly into analogical tendency when subjects walked while wearing two kinds of custom-made insoles. To prevent imbalance caused by the higher MLA of pes cavus deformities, the body needed more muscle activities than the normal foot condition. The reduction of EMG activity, therefore, signified that custom-made insoles would help to efficiently use muscles, and relieve the burden and fatigue in the lower limbs on gait for a long time [38].

In conclusion, custom-made insoles dispersed the pressure concentrated by the higher MLA of pes cavus deformity. It helped patients to walk by offering support to prevent disease on pes cavus foot, and to relieve fatigue and burden on the lower limbs muscles. In particular, the extension structure in the forefoot was more helpful for support. This study found that the custom-made insoles for pes cavus foot significantly affected the biomechanical movement of lower extremities on gait. The result of these useful analyses will be able to be utilized in the manufacture of functional insoles and lower extremity orthotic devices for individuals with pes cavus. This study, especially, shows that custommade insoles can improve efficient use of muscles in the pes cavus patient.

\section{Acknowledgments}

This work was supported by National Research Foundation of Korea (NRF) grant funded by the Korea government (MSIP) (NRF-2013R1A2A2A04016782) and by Basic Science Research Program through the National Research Foundation of Korea (NRF) funded by the Ministry of Education, Science and Technology (NRF-2012R1A1B3003952).

\section{References}

[1] A.K. Ramanathan, P. Kiran, G.P. Arnold, W. Wang and R.J. Abboud, Repeatability of the pedar-X in-shoe pressure measuring system, Foot and Ankle Surgery 16 (2010), 70-73.

[2] Y. Kim, S. Kim, J. Son and B. Jeong, Kinetic role of the metatarsophalangeal joint in normal walking: Joint moment and power, International Journal of Precision Engineering and Manufacturing 13 (2012), 1481-1485.

[3] T. Marasović, M. Cecić and V. Zanchi, Ground reaction forces in gait: statistical analysis and interpretation, In Proceedings of the 9th WSEAS International Conference on Simulation, Modeling and Optimization (SMO'09) (2009), 108111.

[4] R.A. Donatelli, The Biomechanics of the Foot and Ankle, The F. A. Davis Company, Philadelphia, 1995.

[5] J. Perry, Gait Analysis, SLACK, INC., New Jersey, 1992. 
[6] W. Qassem, Impulse response due to jumping on shoes of various stiffness and damping, Bio-medical Materials and Engineering 13 (2003), 167-180.

[7] S.L. Turek, Turek's Orthopaedics: Principles and Their Application, 5th ed., Williams \& Wilkins, Baltimore, 1994.

[8] J.R. Kim, S.K. Lee and H.S. Lee, First metatarsal dorsal close wedge osteotomy combined with medial cuneiform plantar open wedge osteotomy for the treatment of a cavus foot, Journal of the Korean Orthopaedic Association 45 (2010), 24-30.

[9] M. Walker and H.J. Fan, Relationship between foot pressure pattern and foot type, Foot \& Ankle International 19 (1998), 379-383.

[10] D.A. Brewerton, P.H. Sandifer and D.R. Sweetnam, "Idiopathic" pes cavus, British Medical Journal 2 (1963), 659.

[11] L. Paulos, S.S. Coleman and K.M. Samuelson, Pes cavovarus, The Journal of Bone \& Joint Surgery 62 (1980), $942-953$.

[12] M. Sabir and D. Lyttle, Pathogenesis of pes cavus in charcot-marie-tooth disease, Clinical Orthopaedics and Related Research 175 (1983), 173-178.

[13] I.J. Alexander and K.A. Johnson, Assessment and management of pes cavus in charcot-marie-tooth disease, Clinical Orthopaedics and Related Research 246 (1989), 273-281.

[14] R. Sanders, P. Fortin, T. DiPasquale and A. Walling, Operative treatment in 120 displaced intraarticular calcaneal fractures results using a prognostic computed tomography scan classification, Clinical Orthopaedics and Related Research 290 (1993), 87-95.

[15] Z. Azmaipairashvili, E.C. Riddle, M. Scavina and S.J. Kumar, Correction of cavovarus foot deformity in charcot-marietooth disease, Journal of Pediatric Orthopaedics 25 (2005), 360-365.

[16] M.G. Benedetti, F. Catani, F. Ceccarelli, L. Simoncini, S. Giannini and A. Leardini, Gait analysis in pes cavus, Gait Posture 5 (1997), 169.

[17] A.H. Franco, Pes cavus and pes planus analyses and treatment, Physical Therapy 67 (1987), 688-694.

[18] J. Burns, J. Corsbie, A. Hunt and R. Ouvrier, The effect of pes cavus on foot pain and plantar pressure, Clinical Biomechanics 20 (2005), 877-882.

[19] D.M. Brody, Techniques in the evaluation and treatment of the injured runner, The Orthopedic Clinics of North America 13 (1982), 541-558.

[20] R.D. D'Ambrosia, Orthotic devices in running injuries, Clinics in Sports Medicine 4 (1985), 611-618.

[21] V. Oleksik, A. Pascu, C. Deac, R. Fleaca and M. Roman, Experimental strain field distribution in ankle-foot orthosis (AFO), In Proceedings of the 2nd WSEAS International Conference on Biomedical Electronics and Biomedical Informatics (2009), 94-98.

[22] B.M. Nigg, W. Herzog and L.J. Read, Effect of viscoelastic shoe insoles on vertical impact forces in heel-toe running, The American Journal of Sports Medicine 16 (1988), 70-76.

[23] G.P. Brown, R. Donatelli, P.A. Catlin and M.J. Wooden, The effect of two types of foot orthoses on rearfoot mechanics, Journal of Orthopaedic \& Sports Physical Therapy 21 (1995), 258-267.

[24] E. Cahntelau and P. Haage, An audit of cushioned diabetic footwear: Relation to patient compliance, Diabetic Medicine 11 (1994), 114-116.

[25] J.J. Wertsch, L. Frank, H. Zhu, M.B. Price, G.F. Harris and H.M. Alba, Plantar pressures with total contact casting, Journal of Rehabilitation Research and Development 32 (1995), 205-205.

[26] M.E. Edmonds, Progress in care of the diabetic foot, Lancet 354 (1999), 270-272.

[27] S. Albert and C. Rinoie, Effect of custom orthotics on plantar pressure distribution in the pronated diabetic foot, The Journal of Foot and Ankle Surgery 33 (1993), 598-604.

[28] C. Windle, S. Gregory and S. Dixon, The shock attenuation characteristics of four different insoles when worn in a military boot during running and marching, Gait \& Posture 9 (1999), 31-37.

[29] J.H. Kang, M.D. Chen, S.C. Chen and W.L. His, Correlations between subjective treatment responses and plantar pressure parameters of metatarsal pad treatment in metatarsalgia patients: a prospective study, BMC Musculoskeletal Disorders 7 (2006), 95.

[30] M.J. Mueller, D.J. Lott, M.K. Hastings, P.K. Commean, K.E. Smith and T.K. Pilgram, Efficacy and mechanism of orthotic devices to unload metatarsal heads in people with diabetes and a history of plantar ulcers, Physical Therapy 86 (2006), 833-842.

[31] J.Y. Jung, J.H. Kim, K. Kim, P.H. Trieu, Y.G. Won, D.K.Kwon and J.J. Kim, Evaluation of insole-equipped ankle foot orthosis for effect on gait based on biomechanical analysis, Korean Journal of Sport Biomechanics 20 (2010), 469-477.

[32] J.K. Choi, I.S. Park, H.J. Lee, Y.G. Won and J.J. Kim, Evaluation for biomechanical effects of metatarsal pad and insole on gait, Korean Journal of Sport Biomechanics 20 (2011), 487-494.

[33] T. Ryu, H. Soon Choi, H. Choi and M.K. Chung, A comparison of gait characteristics between Korean and western people for establishing Korean gait reference data, International Journal of Industrial Ergonomics 36 (2006), 1023-1030. 
[34] S.Y. Park, S.Y. Lee, H.C. Kang and S.M. Kim, EMG analysis of lower limb muscle activation pattern during pedaling: Experiments and computer simulations, International Journal of Precision Engineering and Manufacturing 13 (2012), 601-608.

[35] E.H. Kim, H.K. Cho, T.W. Jung, S.S. Kim and J.W. Chung, The biomechanical evaluation of functional insoles, Korean Journal of Sport Biomechanics 20 (2010), 345-353.

[36] C.H. Yu, S.H. Shin, H.C. Jeong, D.Y. Go and T.K. Kwon, Activity analysis of trunk and leg muscles during whole body tilt exercise, Bio-Medical Materials and Engineering 24 (2014), 245-254.

[37] W.P. Chen, C.W. Ju and F.T. Tang, Effects of total contact insoles on the plantar stress redistribution: A finite element analysis, Clinical Biomechanics 18 (2003), S17-S24.

[38] C.J. Snijders, P.F. Hermans, R. Niesing, C.W. Spoor and R. Stoeckart, The influence of slouching and lumbar support on iliolumbar ligaments, intervertebral discs and sacroiliac joints, Clinical Biomechanics 19 (2004), 323-329. 